\title{
Parylene Neurocages for Electrical Stimulation on Silicon and Glass Substrates
}

\author{
Angela Tooker, Jon Erickson, Gary Chow, Yu-Chong Tai, and Jerry Pine
}

\begin{abstract}
We present a refined method and design for building parylene neurocages for in vitro studies of live neural networks. Parylene neurocages are biocompatible and very robust, making them ideally suited for studying the synaptic connections between individual neurons to gain insight into learning and memory. The neurocage fabrication process incorporates electrodes into the neurocages to allow for stimulation and recording of action potentials. These neurocages can be fabricated on either silicon or glass substrates. The resulting neurocages have a long term cell survival rate of $\sim 50 \%$, and have proven to be $\mathbf{9 9 \%}$ effective in trapping neurons. Preliminary results demonstrate that current pulses passing through the electrode can stimulate action potentials in the neurons trapped in neurocages.
\end{abstract}

\section{INTRODUCTION}

$\mathrm{N}$ EURONS play an important role in many of our biological and cognitive functions. Many studies concentrate on the properties of neurons and the neural networks they form; unfortunately, it is difficult to study these networks in vivo. Initial in vitro techniques used patterned extracellular electrode arrays [1], [2], but neuron mobility and lack of neuron-to-electrode specificity limit the use of these arrays, especially in long-term studies. Our strategy counteracts this difficulty by using micromachined structures to physically trap individual neurons in close proximity to electrodes, without inhibiting their growth.

The first implementation was the neuro-well, a bulk micromachined silicon device that allows neural networks to form, while trapping individual neurons in close proximity to electrodes [3], [4]. Action potentials were recorded in these early devices with a typical SNR of about 50:1 [4]. Unfortunately, the fabrication and scaling complexities of these devices limited their continued development.

To address these problems, our group developed surface micromachined parylene neurocages [5]-[8]. The initial

Manuscript received April 3, 2006. This work was supported by the NIH under Award Number R01 NSO44134.

A. Tooker is with the Department of Electrical Engineering, California Institute of Technology, Pasadena, CA 91125, USA (phone: 626-395-3367; fax: 626-584-9104; e-mail: atooker@mems.caltech.edu).

J. Erickson is with the Department of Bioengineering, California Institute of Technology, Pasadena, CA 91125, USA (e-mail: erickson@caltech.edu).

G. Chow is with the Department of Bioengineering, California Institute of Technology, Pasadena, CA 91125, USA (e-mail: gangc@caltech.edu).

Y. C. Tai is with the Department of Electrical Engineering, California Institute of Technology, Pasadena, CA 91125, USA (e-mail: yctai@mems.caltech.edu).

J. Pine is with the Department of Physics, California Institute of Technology, Pasadena, CA 91125, USA (e-mail: jpmail@caltech.edu). neurocage design [5], [6] achieved some neuron outgrowth, but long-term cell survival was low $(<25 \%)$. In addition, approximately $10 \%$ of the neurons escaped from these neurocages. Subsequent neurocage designs [7], [8] increased the long-term cell survival rate to $>50 \%$ and decreased the escape rate to $<1 \%$.

The parylene neurocage design presented here incorporates electrodes into the neurocages, to allow for stimulation and recording of action potentials. The fabrication process used to create these new neurocages is flexible, allowing fabrication on both silicon and glass substrates.

\section{Methodology}

\section{A. Design}

The parylene neurocage consists of a chimney, $30 \mu \mathrm{m}$ in diameter and $4 \mu \mathrm{m}$ high, with a $20 \mu \mathrm{m}$ inlet hole at the top for loading neurons (Fig. 1). Extending out from the chimney are 6 tunnels, for neuron outgrowth, interleaved with 6 anchors, for mechanical stability. The tunnels are $1 \mu \mathrm{m}$ high, $10 \mu \mathrm{m}$ wide, and $25 \mu \mathrm{m}$ long. Each neurocage has its own electrode in the center of the chimney, $10 \mu \mathrm{m}$ in diameter.

The neurocage array $(4 \times 4)$ consists of 16 neurocages, each designed to hold a single neuron, centered within a $440 \mu \mathrm{m} x$ 440 $\mu \mathrm{m}$ square (Figs. 2-3).

Parylene was chosen to be the structural material in this

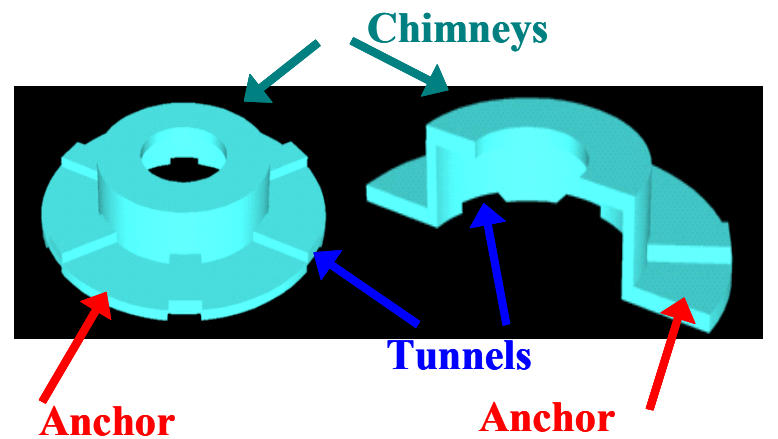

Fig. 1. 3D neurocage model, showing tunnels, anchors, and chimneys.

application because it is biocompatible, non-toxic, extremely inert, and resistant to moisture and most chemicals. Hence, parylene is well suited for long-term cell culture experiments. Its conformal deposition makes it easy to fabricate three-dimensional structures like the neurocage. In addition, parylene is transparent; thus when neurons are 


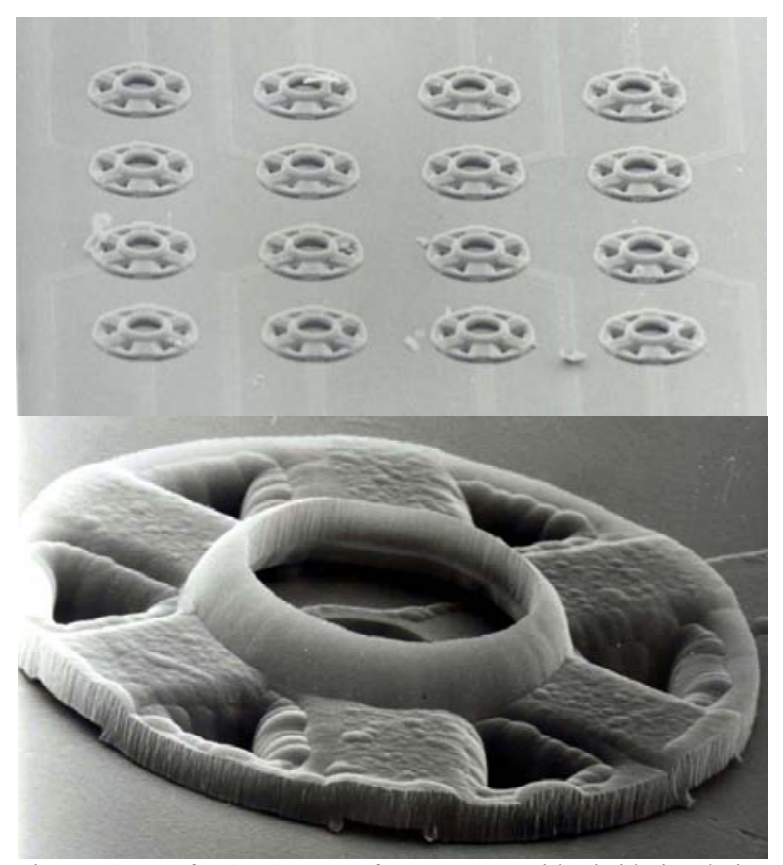

Fig. 2. SEM of a 4 x 4 array of neurocages with nitride insulation (top). SEM of a neurocage with parylene insulation (bottom).

loaded into neurocages, they can easily be seen.

\section{B. Fabrication}

The neurocages are fabricated by first growing a thin layer of oxide $(500 \mathrm{~nm})$ silicon substrate (not necessary for glass substrates). The chrome-gold for the electrodes is thermally evaporated and patterned. The insulation layer, either parylene $(2 \mu \mathrm{m})$ or silicon nitride $(1 \mu \mathrm{m})$, is then deposited. Openings for the electrodes are etched using either $\mathrm{O}_{2}$ plasma (for parylene) or $\mathrm{SF}_{6}$ plasma (for silicon nitride).

Anchors for the neurocage on silicon are then etched through the insulation layer and into the silicon substrate using a DRIE process developed in our group for mechanically securing parylene to a substrate [9]. The DRIE uses a modified Bosch process: 60 loops of a standard Bosch process to make an anisotropic trench with nearly vertical sidewalls, and a subsequent 30 -second $\mathrm{SF}_{6}$ isotropic etch to create a mushroom-like bottom. The anchors are approximately $50 \mu \mathrm{m}$ deep.

As the standard Bosch process for the DRIE does not etch glass, the neurocages on glass require a different anchoring method. After the electrodes are patterned, a thin parylene layer $(0.5 \mu \mathrm{m})$ is deposited and then baked at $350^{\circ} \mathrm{C}$ for 2 hours in a $\mathrm{N}_{2}$ atmosphere. This melts and hardens the parylene on the glass substrate. The anchors for the neurocage are then etched into the baked parylene layer and the glass is etched out from underneath with HF. This creates a short mushroom-like structure, similar to that created by the DRIE on the silicon substrate.

Next, $1 \mu \mathrm{m}$ of aluminum is thermally evaporated and patterned to form the tunnels. If parylene is used as the insulation layer, a thin layer of aluminum $(200 \mathrm{~nm})$ is

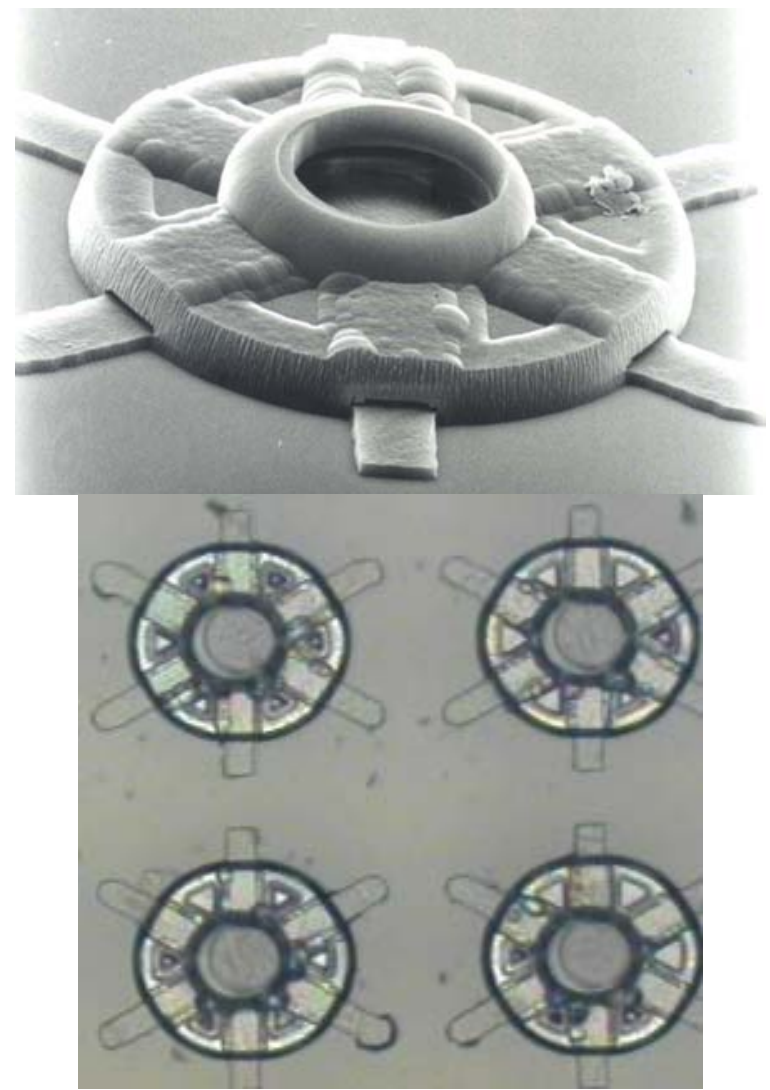

Fig. 3. SEM of a neurocage on glass (top). Optical picture of neurocages on glass (bottom).

thermally evaporated as an etch stop to protect the parylene insulation. A $4 \mu \mathrm{m}$ thick layer of photoresist is patterned to create the chimneys.

Subsequently, a single layer of parylene $(4 \mu \mathrm{m})$ is deposited. The chimneys are etched open using the DRIE process developed in our group [10]. A combination of $\mathrm{O}_{2}$ and $\mathrm{C}_{4} \mathrm{~F}_{8}$ plasmas are used to create vertical sidewalls. $\mathrm{O}_{2}$ plasma is then used to etch open the tunnels.

Finally, the sacrificial aluminum and photoresist for the tunnels and chimneys is removed with aluminum etchant and acetone. The chips are then glued to a carrier and the electrodes are platinized.

\section{Cell Culture}

After sterilization with UV light, the neurocages are covered with warm $\left(37^{\circ} \mathrm{C}\right) 0.05 \%$ PEI solution in borate buffer solution for 12 hours, which penetrates the neurocages, including the tunnels. The PEI is subsequently rinsed out, and $2 \mathrm{uM}$ laminin is added. After overnight incubation, the laminin is rinsed out and the neurocages are ready for culture. Neurons are plated at a density of $30 \mathrm{~K} / \mathrm{cm}^{2}$. 
For neurocages on a silicon substrate, cells are loaded manually into the neurocages with a pressure-driven micropipette.

For neurocages on a glass substrate, cells are loaded using a laser tweezers system consisting of an inverted microscope, a $1064 \mathrm{~nm}$ or $980 \mathrm{~nm}$ laser module, a beam expander, a motorized mechanical stage, a CCD camera, and steering mirrors. The system uses a single, tightly focused laser beam to trap a neuron and lift it into the neurocage.

The first signs of neuron growth usually appear within 1224 hours of loading.

\section{RESUlTS AND DISCUSSION}

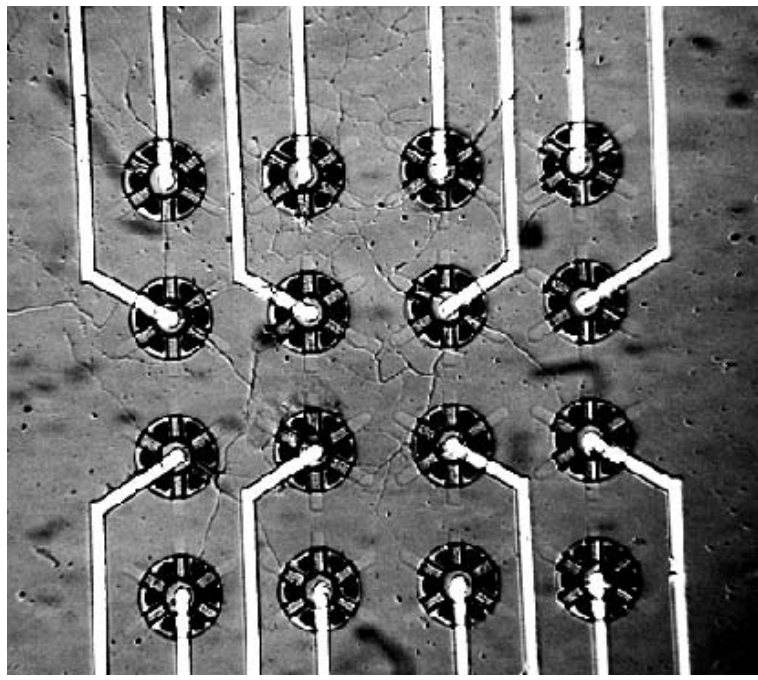

Fig. 4. Nomarski photo of a neurocage culture, 8 days in vitro. Neuronal processes are seen extending through the tunnels and networking with other trapped neurons.

Successful growth of live neural networks has been achieved using $4 \times 4$ arrays of neurocages on silicon, with both parylene and silicon-nitride insulation (Figs. 4-5). As can be seen, the neuronal processes extend through the tunnels and network with neurons in other neurocages. The cultured neurons remain viable and trapped, with virtually

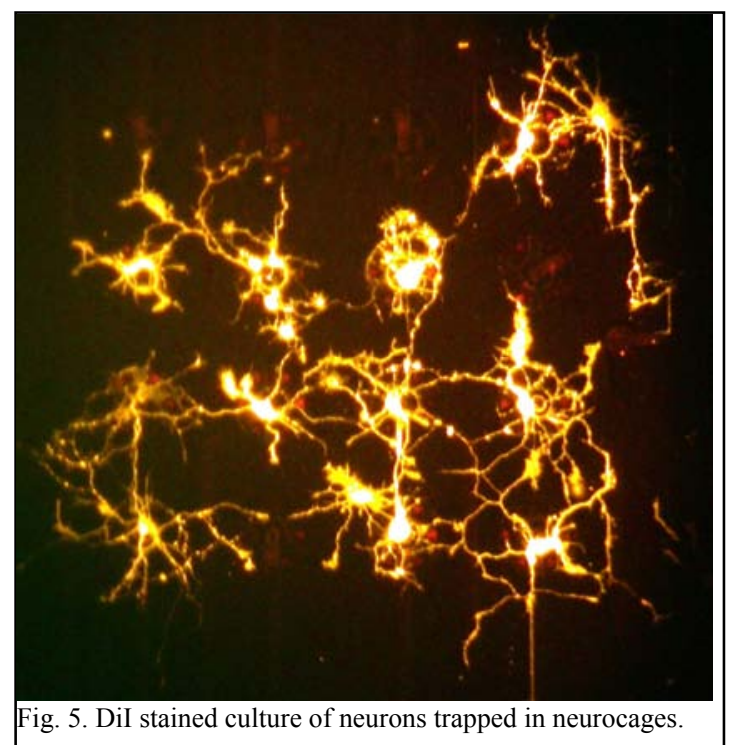

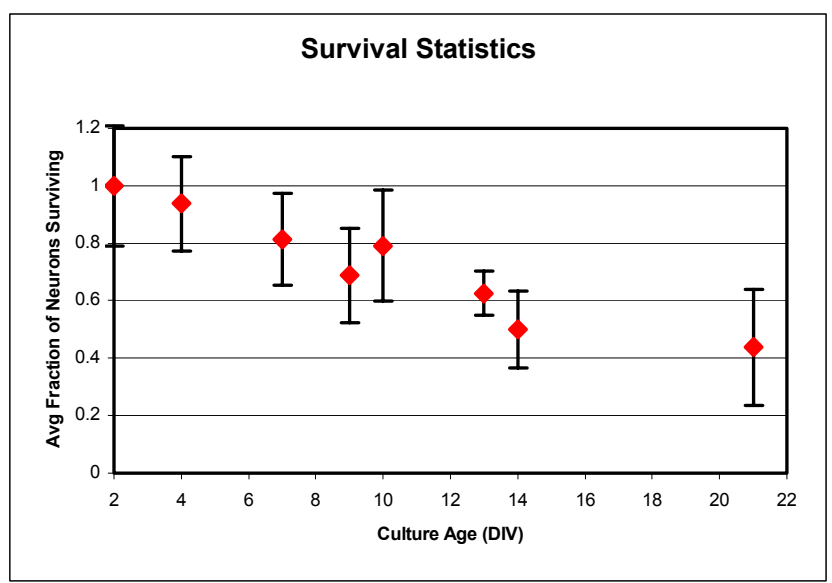

Fig. 6. Survival statistics for neurons grown in neurocages with parylene insulation, both with and without platinized electrodes.

no escape, from the neurocages. The survival rate for these neurons is $\sim 50 \%$ after 2 weeks in vitro (Fig. 6). No significant difference has been noted among the survival rates in neurocages with parylene or silicon nitride insulation. While this survival rate is promising and allows for stimulation and recording from the neural network, methods for increasing the long-term survival rate of the neurons, such as modifications of the neurocage dimensions or design, should be explored. Preliminary results, however, demonstrate that current pulses passing through the electrode can stimulate action potentials in the neurons trapped in neurocages.

Neurons have been successfully placed into the neurocages on glass using the laser tweezers (Fig. 7).

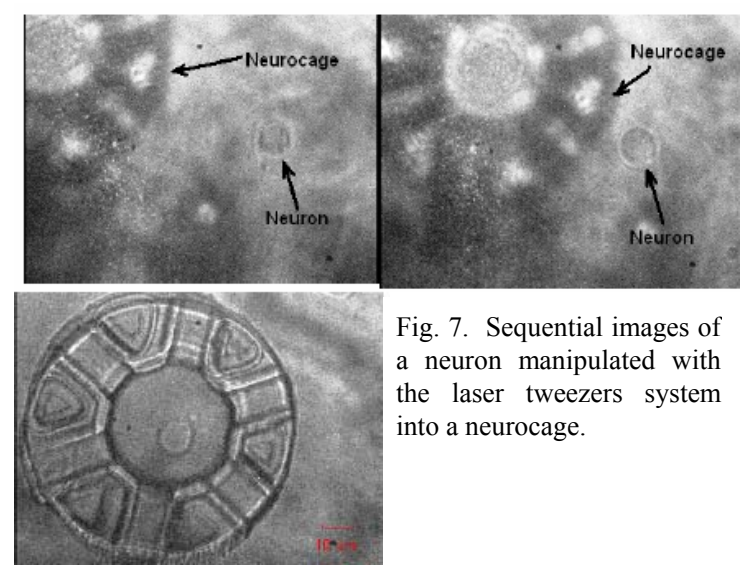

\section{ACKNOWLEDGMENT}

We would like to thank Mr. Trevor Roper for assistance with fabrication and testing and Mr. Pat Koen for assistance with obtaining the SEMs.

\section{REFERENCES}

[1] J. Pine, "Recording action potentials from cultured neurons with extracellular microcircuit electrodes," Journal of Neuroscience Methods, 2, pp. 19-31, 1980. 
[2] Y. Jimbo, T. Tateno, and H.P.C. Robinson, "Simultaneous induction of pathway-specific potentiation and depression in networks of cortical neurons," Biophysical Journal, vol. 76, pp. 670-678, Feb. 1999.

[3] J.A. Wright, S.T. Lucic, Y.C. Tai, M.P. Maher, H. Dvorak, and J. Pine, "Towards a functional MEMS neurowell by physiological experimentation," ASME International Mechanical Engineering Congress and Exposition, DSC-Vol. 59, Atlanta, GA, pp. 333-338, Nov. 1996.

[4] M.P. Maher, J. Pine, J. Wright, and Y.C. Tai, "The neurochip: a new multielectrode device for stimulating and recording from cultured neurons," Journal of Neuroscience Methods, 87, pp. 45-56, 1999.

[5] Q. He, E. Meng, Y.C. Tai, C.M. Rutherglen, J. Erickson, and J. Pine, "Parylene neuro-cages for live neural networks study," Transducers, pp. 995-998, 2003.

[6] E. Meng, Y.C. Tai, J. Erickson, and J. Pine, "Parylene technology for mechanically robust neuro-cages," MicroTAS 2003, pp. 1109-1112.

[7] A. Tooker, J. Erickson, Y.C. Tai, and J. Pine, "Robust and biocompatible neurocages," MicroTAS 2004, Malmo, Sweden, pp. 6466.

[8] A. Tooker, E. Meng, J. Erickson, Y.C. Tai, and J. Pine, "Biocompatible parylene neurocages," IEEE Engineering in Medicine and Biology, vol. 24, no. 6, pp. 30-33, Nov./Dec. 2005.

[9] M. Liger, D.C. Rodger, and Y.C. Tai, "Robust parylene-to-silicon mechanical anchoring", $16^{\text {th }}$ IEEE International Conference on Micro Electro Mechanical Systems, MEMS '03, Kyoto, Japan, pp. 602-605, Jan. 2003.

[10] E. Meng and Y.C. Tai, "Parylene etching techniques for microfluidics and bioMEMS," Proceedings, The $18^{\text {th }}$ International Conference on Micro Electro Mechanical Systems, MEMS '05, Florida, USA, pp. 568-571, Jan. 2005. 